\title{
Competencia de Sicyos polyacanthus en CAÑa de AzÚCAR ${ }^{1}$
}

\author{
Interference of Sicyos polyacanthus in Sugarcane \\ CHAILA, S. ${ }^{2}$, DIAZ, L. ${ }^{2}$, SOBRERO, M.T. ${ }^{3}$ y PISCITELLI, F.R. ${ }^{2}$
}

\begin{abstract}
RESUMEN - Se realizó este ensayo con el objeto de medir los efectos de la competencia permanente y especifica de S. polyacanthus sobre el cultivar CP 74-2005 en la localidad de Monte Rico ( $27^{\circ} 21^{\prime} \mathrm{S}-65^{\circ} 36^{\prime} \mathrm{W}$, Tucumán-Argentina). El diseño experimental, completamente aleatorizado, se estableció en un cañaveral infestado naturalmente. Las parcelas fueron de $64 \mathrm{~m}^{2}$ con cuatro surcos de diez metros de largo. Los efectos de la competencia fueron medidos en tres niveles de infestación: Alta (superior a $5 \mathrm{pl} \mathrm{m}^{-2}$ ), Media $\left(1-4 \mathrm{pl} \mathrm{m}^{-2}\right)$ y Baja (inferior a $1 \mathrm{pl} \mathrm{m}^{-2}$ ). Las mediciones se efectuaron sobre los dos surcos centrales de la parcela; para altura se analizó el promedio de diez mediciones por infestación y por repetición; y para producción de caña y de azúcar se trabajó con una muestra de caña por parcela, extraída del mismo sitio. Los efectos de la maleza en distinto grado de infestación sobre la altura del cañaveral fueron altamente significativos respecto del testigo obteniéndose pérdidas del $48,58 \%$ en baja infestación. En el número de tallos se encontraron pérdidas significativas en los diferentes niveles llegando a $61,87 \%$ en infestación alta. En la producción de caña por hectárea se produjeron perdidas de $43,89 \%$ en infestación media, mientras que en la producción de azúcar se registraron perdidas de 42,28 y 20,38\% en infestación alta y baja respectivamente.
\end{abstract}

Palabras claves: maleza, Cucurbitaceae, interferencia, Saccharum spp.

ABSTRACT - This work aimed to measure the effects of permanent and specific interference of S. polyacanthus on CP 74-2005 sugarcane cultivar in Monte Rico (27 $21^{\circ}$ 'S- $65^{\circ} 36^{\prime} \mathrm{W}$ ) (Tucumán province, Argentina). A complete randomized experimental design was carried out on a naturally infested sugarcane field. Each $64 \mathrm{~m}^{2}$-plot consisted of four ten-meter long furrows. The effects

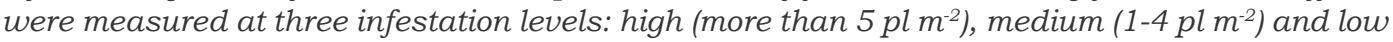
(less than $1 \mathrm{pl} \mathrm{m}^{-2}$ ). The measurements were taken on two central furrows in each plot. Height was taken by means of the average of ten measurements per infestation and per replication. Crop yield was evaluated by means of one sugarcane sample per plot, at the same place. The interference effects on height at different infestation degrees were highly significant in relation to the control, reaching $48.58 \%$ of losses in low infestation. In high infestation, there were significant losses of stem number, at the different levels, reaching $61.87 \%$. Sugarcane yield per hectare presented a $43.89 \%$ loss under medium infestation. Sugar production presented $42.28 \%$ and $20.38 \%$ of losses in high and low infestation, respectively.

Key words: $\quad$ weed, Cucurbitaceae, interference, Saccharum spp.

Recebido para publicação em 14.8.2003 e na forma revisada em 17.12.2003.

Profesores e Investigadores Facultad de Agronomía y Zootecnia. Cátedra de Caña de Azúcar, Universidad Nacional de Tucumán, Av. Roca 1900, 4000 San Miguel de Tucumán, Argentina. Tel. 0054381. 4364147. FAX: 0054381- 4364156, <sach@manant.unt.edu.ar>. ${ }^{3}$ Docente-investigador Cátedra de Matología. Universidad Nacional de Santiago del Estero, Av. Belgrano 1912, 4200 Santiago del Estero, Argentina. 


\section{INTRODUCCION}

S. polyacanthus (Cucurbitaceae) entra en competencia con el cultivo de caña de azúcar al crecer y convivir, dentro de las cepas, en la primavera y el verano. Ruderal y originaria de regiones selváticas, ha logrado adaptarse al cultivo, y encuentra en él condiciones más propicias que en su hábitat natural. Ello la convierte, al presente, en la única maleza latifoliada y anual que puede ocasionar serios problemas en los cañaverales de Tucumán, Argentina (Chaila, 1997b).

Son pocos los trabajos de investigación y experimentales que existen en Argentina, sobre competencia especifica de malezas en caña de azúcar (Arévalo et al., 1974); (Arévalo et al., 1977b); (Arévalo et al., 1977a); (Cerrizuela et al., 1983); (Chaila, et al., 2002).

Según Chaila et al., (1997), muchas son las teorías que se enunciaron acerca de la supervivencia de Sicyos. Una es la asociación con el cultivo de caña de azúcar, y conceptualmente se basa en: a) La especie es 2,4-D resistente. b) Siempre se usó herbicidas inadecuados para su control. c) Entre las cepas de la caña no resulta efectivo el control. d) Normalmente entre las lineas (trochas) se efectúa control mecánico, y no químico. e) Los procedimientos de cosecha mecánica e integral aseguran su dispersión. f) El habitat para su establecimiento es el que le proporcionan las cepas y sus tallos, donde puede trepar y desarrollarse.

La cantidad de tallos del cañaveral depende de tres factores importantes, que condicionarán la actividad perjudicial de la maleza: a) Rápido cierre del cañaveral. b) Ancho de cepas. c) Abundancia del follaje. Pero lo único que puede explicar los efectos de vuelco del cañaveral y la excesiva cobertura de la maleza, es un análisis estructural que, mediante sus indices, determine la incidencia de la baja densidad de la especie en el desarrollo de las poblaciones compartidas (Chaila \& Cerrizuela, 1993).

Autores como Cousens, 1991; De Wit, 1961; Roush et al., 1989, Peterson, 1989, establecieron que las densidades de plantas de proporciones variables son utilizadas para determinar la existencia de una competencia relativa entre dos especies.
Las malezas pueden germinar antes o después de la emergencia del cultivo de caña de azúcar y esto determinará el sistema de manejo apropiado para el óptimo control (Chaila, 1997a). La meta de los estudios de competencia entre maleza-cultivo es la determinación del período de tiempo, durante el ciclo del cultivo, en el cual reducen el rendimiento y afectan la calidad por competencia entre las plantas (Strahan et al., 2000). Para Santos et al., (1998), la habilidad competitiva de la especie está sugerida por el incremento de una especies sobre otra respecto a la variable rendimiento.

Arévalo (1977a) determinó que las pérdidas por competencia dependen de varios factores, entre los que menciona los ecofisiológicos y los dependientes del desarrollo económico y tecnológico.

Rochecouste (1967) estableció que la caña de azúcar es muy sensible a la competencia de malezas en los primeros estadios de crecimiento y se extiende hasta que completa el macollaje y el sistema radical en el segundo estadio.

Existen trabajos de competencia específica de Rotboellia cochinchinensis sobre caña de azúcar, efectuado por Lencse \& Griffin (1991) en Louisiana, que registraron perdidas del 43\% en el rendimiento. Según Sharma \& Zelaya (1986), la misma especie, cuando no es controlada ocasiona en el cultivo de maíz $80 \%$ de reducción en los rendimientos.

Kuva et al., (2001) estudiando la competencia especifica y permanente de Brachiaria decumbens encontraron que ésta, produce una reducción del $82 \%$ de la producción del cultivo.

Los antecedentes de especies de Sicyos como competidora de un cultivo son pocos. Se menciona a $S$. angulatus como maleza competidora del cultivo de maíz en Italia (Rapparini, 1999), y como maleza presente en varios cultivos de Turquía (Terzioglu \& Ansin, 1999).

No se conocen en la actualidad los efectos competitivos de $S$. polyacanthus sobre los cañaverales y los cultivares empleados.

El propósito de estos estudios fue evaluar la competencia específica y permanente de S. polyacanthus sobre el cultivar de caña de azúcar CP 74-2005. 


\section{MATERIAL Y METODOS}

El ensayo experimental, completamente aleatorizado, se implantó en octubre del año 2000, en la localidad de Monte Rico, Departamento de Monteros, Tucumán, Argentina $\left(27^{\circ} 21^{\prime} \mathrm{S}-6^{\circ} 36^{\prime} \mathrm{W}\right)$, el clima es subtropical (templado-cálido-húmedo), situado en la zona del piedemonte con suelos francos a francoarenosos sin grava, pertenece al grupo de los hapludoles cumúlicos con un pH de 6,5 y un contenido de materia orgánica de 3,5\%, con lluvias de $950 \mathrm{~mm}$ anuales, invierno seco con heladas ocasionales y verano lluvioso, la temperatura media anual es de $19{ }^{\circ} \mathrm{C}$. E1 cañaveral seleccionado, estaba compuesto de cañas socas de 5 años y se encontraba infestado naturalmente por la maleza. Las parcelas tenian 4 surcos de ancho por 10 metros de largo, distanciados 1,60 metros uno de otro, configurando una superficie de $64 \mathrm{~m}^{2}$. La experiencia se realizó en un solo cultivar: CP 74-2005 en competencia permanente con la maleza.

Los tratamientos fueron:

T1: Alta Infestación

T2: Infestación Media

T3: Baja Infestación

T4: Testigo Limpio, Sin Competencia

Se efectuaron tres repeticiones para cada tratamiento. Se buscó la especificidad de competencia con tratamientos quimicos graminicidas en dosis usuales $(2,4-\mathrm{D}$, 1264 g e.a. ha ${ }^{-1}$ Ametrina 50, 1500 g i.a. ha-1), y deshierbes manuales. En el testigo limpio se realizaron labores cada diez dias con herbicidas y controles mecánicos.

Para los grados de infestación se consideraron las siguientes densidades de $S$. polyacanthus (Chaila \& Cerrizuela, 1993) (Chaila et al., 1997):

a) Alta: más de $5 \mathrm{pl} \mathrm{m}$.2.

b) Media: de 1 a $4 \mathrm{pl} \mathrm{m}$.2.

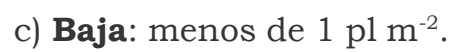

Las mediciones se efectuaron sobre los dos surcos centrales de cada parcela. Se evaluaron los siguientes parámetros en el cultivo: altura de tallos, número de tallos y producción de caña ha $\mathrm{h}^{-1} \mathrm{y}$ azúcar ha- $\mathrm{h}^{-1}$ La altura fue medida antes de la cosecha desde el entrenudo basal en el suelo hasta la hoja +1 (ligula y aurícula completamente desarrollada o primera hoja expandida), se tomó el promedio de diez mediciones por infestación y por repetición. Para producción de azúcar se trabajó con una muestra de 20 cañas por parcela, sobre la cual se efectuaron los análisis de laboratorio (extracción de jugo y determinaciones de brix, pol y pureza).

Los datos obtenidos en los diferentes niveles de competencia fueron sometidos a análisis de la varianza. Las comparaciones entre medias fueron realizadas mediante la Prueba de Tukey al 0,05 de probabilidad.

\section{RESULTADOS Y DISCUSIÓN}

El criterio empleado para medir los efectos de S. polyacanthus sobre el cultivo de caña de azúcar, se basó en un principio de dominio de la especie sobre otras y en el establecimiento de áreas naturales de diferentes grados de infestación (Chaila \& Cerrizuela, 1993) (Chaila et al., 1997) ya definido por otros autores como Cousens (1991); De Wit, (1961); Roush et al., (1989), Peterson, (1989), pero esta maleza posee características diferenciales respecto de otras malezas, en especial en lo que hace a la densidad de competencia.

El número de tallos de caña de azúcar y su altura son algunos de los componentes del rendimiento afectados por la competencia de S. polyacanthus. En la Tabla 1 se observan las diferencias existentes entre las poblaciones de tallos para los distintos niveles de infestación. Existen diferencias estadísticas significativas entre el testigo limpio y los diferentes grados de infestación del cañaveral. En infestación alta, hubo un 68,28\% de reducción de altura y un 48,58\% en infestación baja, con respecto al testigo. En la mayoría de las parcelas de alta y media infestación, los tallos se encontraban en el suelo, el vuelco se produjo por la presión ejercida por la maleza que ha trepado sobre el cañaveral. El menor número de tallos de caña (Tabla 1), se debió a la estricta competencia de la maleza por agua y nutrientes entre octubre y noviembre, donde se produce el macollaje en el cañaveral, y por luz durante el 
Tabla 1 - Efectos de la competencia específica de $S$. polyacanthus sobre altura de plantas, número de tallos y producción de caña y azúcar en función de los diferentes grados de infestación. Tucumán. Argentina, 2000

\begin{tabular}{|c|c|c|c|c|}
\hline \multirow{2}{*}{ Grado de Infestación } & Altura de planta & Número de tallos ha $^{-1}$ & Producción de caña & Producción de azúcar \\
\cline { 2 - 5 } & $(\mathrm{m})$ & $\left(\mathrm{ha}^{-1}\right)$ & \multicolumn{2}{|c|}{$\left(\mathrm{t} \mathrm{ha}^{-1}\right)$} \\
\hline Alta & $1,45 \mathrm{~d}$ & $30,00 \mathrm{~d}$ & $50,30 \mathrm{~d}$ & $5,55 \mathrm{c}$ \\
\hline Media & $2,09 \mathrm{c}$ & $40,67 \mathrm{c}$ & $56,12 \mathrm{c}$ & $5,66 \mathrm{c}$ \\
\hline Baja & $2,35 \mathrm{~b}$ & $54,67 \mathrm{~b}$ & $65,63 \mathrm{~b}$ & $7,66 \mathrm{~b}$ \\
\hline Testigo & $4,58 \mathrm{a}$ & $78,66 \mathrm{a}$ & $84,83 \mathrm{a}$ & $9,62 \mathrm{a}$ \\
\hline CV\% & 2,56 & 6,20 & 2,04 & 5,70 \\
\hline
\end{tabular}

* Las medias seguidas por letras iguales no difieren entre si por la prueba de Tukey al $5 \%$ de probabilidad.

periodo de gran crecimiento del cañaveral, que ocurre desde diciembre en adelante. Como consecuencia de esto, en todos los niveles de infestación, la maleza cubrió al cañaveral trepando sobre él.

Autores como Weiner \& Thomas (1986) y Weiner (1988) explican un suceso similar estableciendo dos hipótesis que recibieron el nombre de competición simétrica y competición asimétrica para la distribución de los recursos entre las plantas competidoras. Hay un efecto de invasión por cobertura de S. polyacanthus sobre el cañaveral, donde recibe luz y al mismo tiempo quita luz al cañaveral.

La única forma de entender la competencia de S. polyacanthus sobre caña de azúcar, es explicándola por la disputa permanente de recursos, desde que la maleza emerge en el mes de octubre en adelante. Sus hábitos de crecimiento le permiten trepar sobre el cultivo y limitar la fotosintesis, produciendo sombra sobre el follaje de la caña afectando el tamaño y produciendo el vuelco del cultivo.

Refiriéndose al cultivo de la caña de azúcar, Brandauer (1977) expresa que la interferencia de malezas afecta principalmente el establecimiento de poblaciones óptimas de tallos por unidad de superficie. La incidencia de una especie sobre el cultivo de caña, desde los primeros estadios de crecimiento, afecta al número de tallos como se experimenta en este trabajo.

En la Tabla 1 también se manifiesta el efecto competitivo de la maleza sobre el número de tallos, según los diferentes grados de infestación. Las pérdidas producidas son $61,87 \%, 48,31 \%$ y $30,51 \%$ para alta, media y baja respectivamente.

$\mathrm{El}$ ancho de la cepa y el rápido cierre son elementos que permitirán al cañaveral reaccionar contra Sicyos y su fitoagresividad; pero la maleza supera a las condiciones del cañaveral, porque este último, no posee agresividad, habilidad competitiva, ni agilidad para expandirse en busca de la luz. Esta especie de maleza efectúa coberturas extensas sobre la parte superior del follaje de las plantas, abarcando varios metros cuadrados; la cobertura será mayor o menor, según sea el momento de aparición de las plántulas y el grado de infestación producida.

La densidad de tallos del cañaveral fue afectada por una densidad muy pobre de la planta nociva. La densidad en la competencia permanente de Sicyos vs Caña no expresa la supremacía de la maleza sobre el cultivo. Esta especie perjudicial utiliza otros elementos que, definiéndolos, nos aproximarían a los conceptos de estructuras poblacionales.

Pese a ser muy pequeñas las densidades de la maleza, siempre habrá respuesta a la competencia; estando esto de acuerdo con lo expresado por García Torres (1991): "en las malas hierbas no es corriente que exista un umbral de no-respuesta del cultivo; incluso con densidades muy bajas de malas hierbas, los rendimientos del cultivo acusan claramente dicha presencia". Pero los efectos de la competencia mínima y los umbrales de $S$. polyacanthus sobre caña no fueron estudiados aún. 
En la Tabla 1 se reproducen los efectos de la competencia específica sobre producción de caña. Existieron diferencias entre los tratamientos, esto quiere decir que, sea cual fuere el grado de infestación, siempre habrá pérdidas o disminución en la producción debida a la infestación de la maleza. Los efectos sobre la producción fueron significativos en todos los niveles, marcándose los números extremos para baja infestación y testigo. Para alta infestación hubo 40,74\% de pérdidas y en baja un $22,64 \%$. Entre la infestación baja, que sería la de menos efectos competitivos y el testigo cultivado, hay $19.205,67 \mathrm{~kg}$ de caña por hectárea. En la Figura 1 se expresan los efectos de la competencia sobre la pérdida de caña para los diferentes tratamientos.

En la Tabla 1 y Figura 3 se establecen las pérdidas en la producción de azúcar según los diferentes grados de infestación. En alta infestación se registraron pérdidas de 42,28\% de azúcar con una producción de 5.552,66 kg azúcar ha ${ }^{-1}$ para alta infestación, contra $9.619,14 \mathrm{~kg}$ azúcar ha ${ }^{-1}$ para el testigo limpio. Se puede identificar la aproximación entre los comportamientos de las infestaciones alta y media, no existiendo diferencias significativas entre su producción. Pero sus valores similares difieren de la producción baja y del testigo cultivado. Con la infestación baja, se obtuvieron valores de producción mayores que con los otros grados de infestación; por consiguiente, las pérdidas manifestadas fueron significativamente menores, lo que no invalida el efecto de $S$. polyacanthus sobre el cultivo. La infestación baja y alta produjeron una merma de producción de 2778,34 y 3907,33 kg de azúcar por hectárea, respectivamente.

Una explicación posible de los efectos perjudiciales ocasionados por esta cucurbitácea en el cultivo de caña, que es de alta eficiencia fotosintética, puede ser compartido con las explicaciones elaboradas por Golberg \& Landa (1991) que basan sus hipótesis en: las respuestas a la habilidad competitiva de una de las especies, la habilidad individual para competir, la abundancia de población, los estadios de crecimiento y el tamaño de las plantas.

Comparando las pérdidas en producción de azúcar con los estudios existentes de competencia específica, se puede afirmar que: S. polyacanthus iguala a las pérdidas ocasionadas por comunidades naturales (Arévalo et al., 1977a), las que alcanzan un $42 \%$ en promedio; supera a las pérdidas que produce Cynodon dactylon (Cerrizuela et al., 1983), con 35,80\% en promedio; y es Sorghum halepense el que supera a S. polyacanthus en pérdidas producidas, con un 76,60\% (Arévalo et al., 1977b). En la competencia especifica de Tithonia tubaeformis con la caña de azúcar se encontró que esta última maleza no supera a Sicyos, ya que en alta infestación produce pérdidas en el número de tallos de 43,26\% y pérdidas en azúcar del 35,03\% (Chaila et al., 2002).

Se concluye que $S$. polyacanthus, en competencia permanente, produce efectos

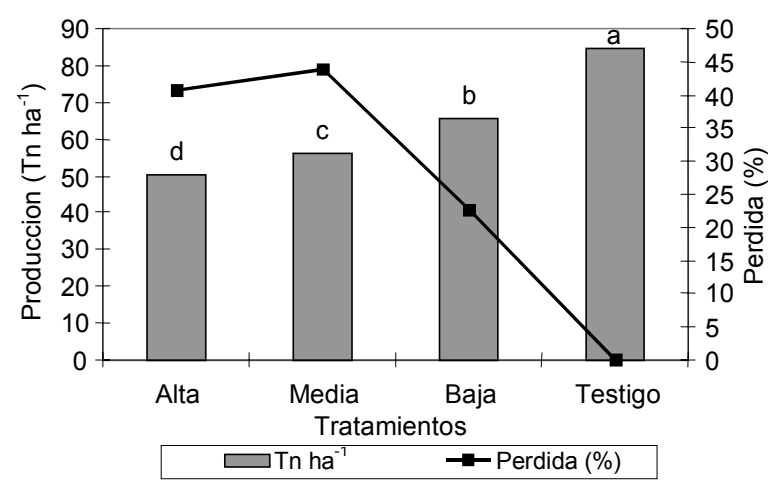

Figura 1 - Producción y pérdida de caña por competencia para diferentes densidades de infestación de la maleza.

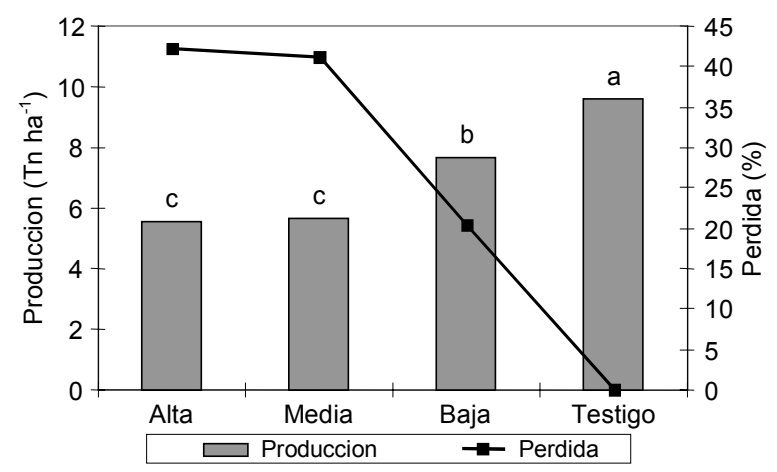

Figura 2 - Producción y perdida de azúcar por competencia en CP 74-2005 para diferentes grados de infestacion de $S$. polyacanthus Cong.

Planta Daninha, Viçosa-MG, v. 22, n. 4, p. 545-551, 2004 
perjudiciales sobre el cultivo de caña de azúcar, que se manifiesta en la disminución del crecimiento de tallos (efectos sobre la altura de plantas), reducción del número de tallos (efectos sobre densidad de cepas), y pérdida importante de producción de caña y de azúcar (efectos sobre el rendimiento).

\section{AGRADECIMIENTOS}

Por la colaboración prestada a los Ingenieros Agrónomos Marcelo H. de la Vega, José M. Fermoselle y Jorge H. Perera. A los Señores Rafael Escobar y Marcelo Pasteris. Al Instituto Cinefotográfico de la Universidad Nacional de Tucumán. Los fondos económicos para la realización de la experimentación fueron aportados por el CIUNT y por el CIME.

\section{LITERATURA CITADA}

ARÉVALO, R. A.; CERRIZUELA, E. A.; SOLDATTI. A. A. Competencia de malezas específicas en caña planta. I. Cyperus rotundus. Malezas y su Control (ASAM), v. 3, n. 1 , p. 59-75, 1974.

ARÉVALO, R. A.; CERRIZUELA, E. A.; OLEA. I. Período crítico de competencia de comunidades naturales de malezas en caña de azúcar. R. Agron. Nor. Argentino, v. 14, n. 1-4, p. 83-99, 1977a.

ARÉVALO, R. A.; CERRIZUELA, E. A.; OLEA. I. Competencia de malezas en caña de azúcar. II. Sorghum halepense (L.) Pers. R. Agron. Nor. Argentino, v. 14, n. $1-4$, p. $39-51,1977 b$.

BRANDAUER, E. A. Relación costo beneficio en el control de malezas en caña de azúcar en Colombia. In: The world of sugar cane. CIBA GEIGY, 1977. p. 52-59.

CERRIZUELA, E. A.; CHAILA, S.; LIZÁRRAGA, A. Respuesta varietal a la competencia de Cynodon dactylon $\mathrm{L}$. (Pers) en caña de azúcar. R. Agron. Nor. Argentino, v. 12, n. 1-4, p. 49-61, 1983.

CHAILA, S. Matoecología de la caña de azúcar. Tucumán: Universidad Nacional de Tucumán, 1997a. 130 p.

CHAILA, S. et al. Efecto de tratamientos mecánicos y químicos sobre la mortalidad y superviviencia de Sicyos polyacanthus Cogn, en caña de Azúcar. ASAPROVE, v. 2, p. 202-209, 1997.

CHAILA, S. Distribución de semillas de Sicyos polyacanthus Cogn. en un suelo cultivado con caña de azúcar. ASAPROVE, v. 2, p. 270-2079, 1997b.

Planta Daninha, Viçosa-MG, v. 22, n. 4, p. 545-551, 2004
CHAILA, S. et al. Competencia permanente de Tithonia tubaeformis (Jacq.) Cass.en caña de azúcar Cv. FAM 8177. Asociación de Biología de Tucumán, Ediciones del Rectorado. Universidad Nacional de Tucumán, 2002. p. 6469

CHAILA, S.; CERRIZUELA, E. A. Interferencias y estrategias de Sicyos polyacanthus Cogn. frente a procesos de alteración poblacional en caña de azúcar. Revista Malezas, v. 3, p. $95-100,1993$.

COUSENS, R. Aspects of the design and interpretation of competition (interference) experiments. Weed. Technol., v. 5, p. 664-773, 1991.

DE WIT, C. T. On competition. Versl. Landbouwk. Onderz., v. 66, p. 1-82, 1961.

GARCIA TORRES, L.; FERNÁNDEZ QUINTANILLA, C. Fundamentos sobre malas hierbas $\mathbf{y}$ herbicidas. Madrid: Mundi Prensa, 1991. 348 p

GOLDBERG, D. E.; LANDA, K. Competitive effect and response: Hierarchies and correlated traits in the early stages of competition. J. Ecol., v. 79, p. 1013-1030, 1991.

KUVA, M. A. et al. Períodos de Interferencia das Plantas Daninhas na Cultura da Cana de Acucar. II.- CapimBraquiária (Brachiaria decumbens). Planta Daninha, v. 19, n. 3, p. 223-330, 2001

LENCSE, R. J.; GRIFFIN, J. L. Itchgrass (Rottboellia cochinchinensis) interference in sugarcane (Saccharum sp.) Weed Technol., v. 8, p. 733-737, 1991.

PETERSON, T. D. A comparison of methods for measuring effects of density and proportion in plant competition experiments. Weed Sci., v. 37, p. 268-275, 1989.

RAPPARINI, G. Weed control in maize and sorghum. Infor. Agrario, v. 55, n. 8, p. 65-82, 1999.

ROCHECOUSTE, E. Weed control in sugarcane. Mauritius, Sug. Ind. Res. Inst. 1967. 117 p.

ROUSH, M. L. et al. A comparison of methods for measuring effects of density and proportion in plant competition experiments. Weed Sci., v. 37, p. 268-275, 1989.

SANTOS, B. M. et al. Phosphorus effects on competitive interactions of smooth pigweed (Amaranthus hybridus) anda common purslane (Portulaca oleracea) withy lettuce (Lactuca sativa). Weed Sci., v. 46, p. 307-312, 1998.

SHARMA, D.; ZELAYA, O. Competition and control of itchgrass (Rottboellia exaltata) in maize (Zea mays). Trop. Pest. Manang, v. 32, p. 101-104, 1986. 
STRAHAN, R. E. et al. Interference between Rotboellia cochinchinensis and Zea maiz. Weed Sci., v. 48, p. 205-211, 2000 .

TERZIOGLU, S.; ANSIN, R. A contribution to exotic plants of Turkey: Sicyos angulatus L. Turkish J. Agric. For., v. 23, n. 3, p. 359-362, 1999.
WEINER, J. Variation in the performance of individuals in plant populations. In: DAVY, HUTCHINGS. ed. Plant population ecology, Oxford: Blackwell Scientific Publications, 1988. p. 559-581.

WEINER, J.; THOMAS, S. C. Size variability and competition in plant monocultures. Oikos, v. 47, p. 211$222,1986$. 